\title{
The influence of music education and training on SLA
}

Barbara Pastuszek-Lipińska

School of English, Adam Mickiewicz University, Poland

https://doi.org/10.36505/ExLing-2008/02/0047/000106

\begin{abstract}
Although music education is one of the human activities that requires the integration of all human senses and the involvement of all cognitive processes: sensory, perceptual, and cognitive learning, memory, emotion, and auditory and motor processes, music has tended to figure only marginally in an approach to second-language acquisition. To explore the extent to which music education influences second-language acquisition musicians and non-musicians were asked to reproduce thrice-repeated sentences in six languages. Musicians outperformed non-musicians in the study. From the results, it appears that the influence of musical expertise extends beyond music processing to speech processing.
\end{abstract}

Key words: music education, second language acquisition

\section{Introduction}

Language and music are universal among human cultures. Both are conveyed by sequences of sounds organized in time, and the temporal or rhythmic aspects are highly important features of both domains. Both domains also involve organized acoustics signals that are used in interpersonal communication, and both involve complex cognitive and motor processes. Thus, it is not surprising that the two domains and the relationship between them have attracted interest of researchers from a variety of disciplines (Magne et al. 2003, Thompson et al. 2004, Jentschke et al. 2005, Lahav et al. 2005, Schellenberg 2005, Magne et al. 2006).

Although the similarities and differences between the two systems have attracted the attention of scientists and researchers from different disciplines for centuries, most of the analyses were conducted during the last three decades, when the issues emerged with a new impetus due to new research methods and technical possibilities. Since then, a fair number of researchers from different domains have examined the issues from different points of view.

\section{Research design \\ Research corpus}

82 word sequences in 6 languages (English: American (15), British English (14), Belgian Dutch (10), French (10), Italian (10), Spanish: European (6) and South American (4) and Japanese (10)) were synthesized for the corpus. The ScanSoft ${ }^{\circledR}$ RealSpeak ${ }^{\mathrm{TM}}$ application was used for this purpose.

ExLing 2008: Proceedings of 2nd Tutorial and Research Workshop on Experimental Linguistics, 25-27 August 2008, Athens, Greece 
Languages were chosen according to their typological classification; among them there were stimuli that included stress-timed, syllable-timed, and morae-timed languages. Among the sequences were questions, statements, and orders.

\section{Participants}

In the reported study participated 106 (53 musicians and 53 non-musicians) non-paid volunteers, Polish native speakers. All subjects were aged from 15 to 69 years, with a mean age of 32 (median 28).

\section{Research procedure}

Participants were asked to reproduce as accurately as they could foreign language sentences - synthetic stimuli after three repetitions with taking into account both segmental (vowels and consonants) and suprasegmental (intonation, rhythm, stress, and rate) features. All subjects' productions were recorded, and, with data gathered from the speakers through special questionnaires, were examined with a battery of tests and analyses.

Additionally, the musical skills of the participating non-musicians were tested (Pastuszek-Lipińska 2003). Data were analysed through several different experiments two of them are shortly presented below.

\section{Experiment 1 - General auditory analysis}

All recordings were rated by the author by an impressionistic auditory analysis. In the first round of data analysis, the scoring procedure was based on a general review and observation of whether all speakers responded to the stimuli and were able to repeat the speech material in the given time and with appropriate accuracy.

\section{Experiment 2 - Listening test 2 - web-based cross-linguistic listening test}

To observe how participants were perceived by native speakers of all languages involved in the study, a web-based experiment with a panel of native speakers of involved languages was designed and conducted.

The group of raters consisted of twenty four native speakers of American English, two native speakers of Belgian Dutch, four native speakers of British English, fifteen native speakers of French, eight native speakers of Italian, three native speakers of Japanese, and nine native speakers of Spanish.

Judges rated subjects' productions in only their mother tongue using the Visual Analogue Scale. 


\section{Research results}

\section{Experiment 1 - results}

Each participant encountered some problems with at least one sentence. However, $65.53 \%$ of musicians' and $46.55 \%$ of non-musicians' productions were rated as correct. So the general analysis revealed better performance of musicians comparing with non-musicians.

\section{Experiment 2 - results}

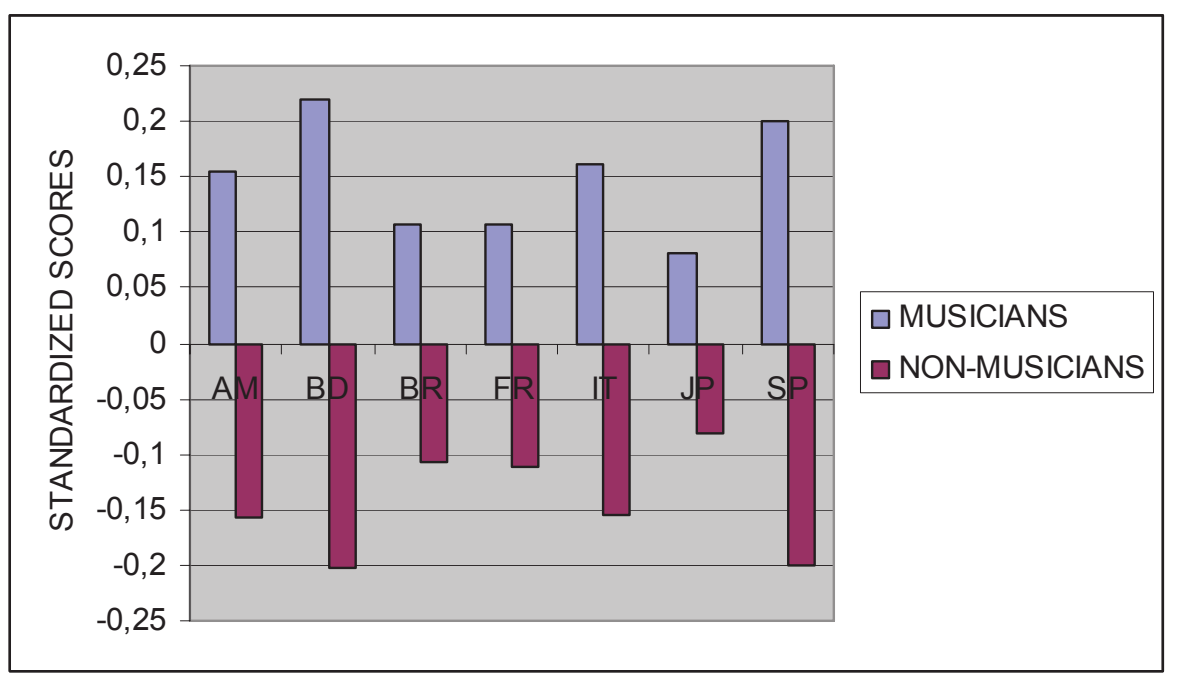

Figure 1. Mean standardized scores obtained by two groups of examinees.

The graphs above present the standardized scores given by the native speakers; it can be seen that in all examined sentences, musicians obtained higher scores (above the average at 0 level), while non-musicians obtained lower scores (below the average).

\section{Conclusions}

Results confirm the hypothesis that musicians would perceive and produce speech sequences better than non-musicians. Moreover, this trend was observed in syllable, stress, and morae-timed languages.

It seems to be clear that even without obvious correlations between variables, the results provide evidence that music education has a significant influence on the acquisition of a second language and more specifically pronunciation.

The results revealed that music education exerted a measurable impact on speech perception and production. Musicians outperformed non-musicians in 
the study. Therefore, the superior performance of the musicians in the task may be interpreted as evidence that music education is an enabling factor in the successful acquisition of a second language.

\section{References}

Jentschke, S., Koelsch, S. and Friederici, A.D. 2005. Investigating the relationship of music and language in children. Influences of musical training and language impairment. In Avanzini, G., Lopez, L., Koelsch, S. and Majno, M. (eds.), Annals of the New York Academy of Sciences, vol. 1060, 231-242, New York, USA.

Lahav, A., Boulanger, A., Schlaug, G. and Saltzman, E. 2005. The power of listening. Auditory-motor interactions in musical training. In Avanzini, G., Lopez, L., Koelsch, S. and Majno, M. (eds.), Annals of the New York Academy of Sciences, vol. 1060, 189-194, New York, USA.

Magne, C., Schön, D. and Besson, M. 2003. Prosodic and melodic processing in adults and children: behavioral and electrophysiologic approaches. In Avanzini, G., Faienza, C., Minciacchi, D., Lopez, L., and Majno, M. (eds.), Annals of the New York Academy of Sciences, vol. 999, 461-476, New York, USA.

Magne, C., Schön, D. and Besson, M. 2006. Musician children detect pitch violations in both music and language better than nonmusician children: Behavioral and electrophysiological approaches. Journal of Cognitive Neuroscience 18(2), 199-211.

Pastuszek-Lipińska, B. 2003. Unpublished test of musical abilities.

Schellenberg, E.G. 2005. Music and cognitive abilities. Current Directions in Psychological Science 14, 322-325.

Thompson, W.F., Schellenberg, E.G. and Husain, G. 2003. Perceiving prosody in speech effects of music lessons. In Avanzini, G., Faienza, C., Minciacchi, D., Lopez, L., and Majno, M. (eds.), Annals of the New York Academy of Sciences, vol. 999, 530-532, New York, USA. 